\title{
Fossils and Plant Evolution: Structural Fingerprints and Modularity in the Evo-Devo Paradigm
}

\author{
Alexandru M.F. Tomescu ${ }^{1}$ and Gar W. Rothwell ${ }^{2}$ \\ ${ }^{1}$ Department of Biological Sciences, Humboldt State University, Arcata, California 95521, USA \\ (+1) 707-826-3229 mihai@humboldt.edu \\ ${ }^{2}$ Department of Environmental and Plant Biology, Ohio University, Athens, Ohio 45701, USA, and \\ Department of Botany and Plant Pathology, Oregon State University, Corvallis, Oregon, 97331, USA \\ (+1) 541-737-5252 rothwell@ohio.edu and rothwelg@oregonstate.edu
}

\begin{abstract}
Fossils constitute the principal repository of data that allow for independent tests of hypotheses of biological evolution derived from observations of the extant biota. Traditionally, transformational series of structure, consisting of sequences of fossils of the same lineage through time, have been employed to reconstruct and interpret morphological evolution. More recently, a move toward an updated paradigm was fueled by the deliberate integration of developmental thinking in the inclusion of fossils in reconstruction of morphological evolution. The vehicle for this is provided by structural fingerprints recognizable morphological and anatomical structures generated by (and reflective of) the deployment of specific genes and regulatory pathways during development. Furthermore, because the regulation of plant development is both modular and hierarchical in nature, combining structural fingerprints recognized in the fossil record with our understanding of the developmental regulation of those structures produces a powerful tool for understanding plant evolution. This is particularly true when the systematic distribution of specific developmental regulatory mechanisms and modules is viewed within an evolutionary (paleo-evo-devo) framework. Here, we discuss several advances in understanding the processes and patterns of evolution, achieved by tracking structural fingerprints with their underlying regulatory modules across lineages, living and fossil: the role of polar auxin regulation in the cellular patterning of secondary xylem and the parallel evolution of arborescence in lycophytes and seed plants; the morphology and life history of early polysporangiophytes and tracheophytes; the role of modularity in the parallel evolution of leaves in euphyllophytes; leaf meristematic activity and the parallel evolution of venation patterns among euphyllophytes; mosaic deployment of regulatory modules and the diverse modes of secondary growth of euphyllophytes; modularity and hierarchy in developmental regulation and the evolution of equisetophyte reproductive morphology. More generally, inclusion of plant fossils in the evo-devo paradigm has informed discussions on the evolution of growth patterns and growth responses, sporophyte body plans and their homology, sequences of character evolution, and the evolution of reproductive systems.
\end{abstract}

Keywords: fossil; morphology; evo-devo; paleobotany; evolution; development; macroevolution; modularity; hierarchy; structural fingerprint; regulatory module; auxin; polar auxin transport; embryophyte evolution; sporophyte evolution; polysporangiophyte; leaf evolution; secondary growth; secondary xylem; vascular cambium; strobilus; Sphenophyta; Equisetum; Lycophyta; root evolution; Lepidodendrales

\section{Fossils are the ultimate evidence for evolution}

Since their earliest occurrences in the fossil record more than 400 million years ago, vascular plants have diversified tremendously. While living species are characterized by a sporophyte that is 
differentiated into a wide array of organs and parts, including stems, leaves, roots, sporangia, seeds, cones, flowers, and fruits, the most ancient vascular plant sporophytes consisted of only simple branching axes with terminal sporangia. The origin of the ancestral tracheophyte body plan and its transition from this simple organization to the complex sporophytes present in most modern tracheophyte lineages were accompanied by numerous dramatic changes in plant structure (Rothwell et al. 2014; Tomescu et al. 2014). Understanding these changes within a developmental framework is key to reconstructing plant evolution and phylogeny, and necessitates in-depth knowledge of both the intermediate stages that populate the different trajectories of evolutionary change, and of the order in which they occurred within each lineage. However, most of these intermediate stages of evolutionary changes that have transformed plants over time are not present among species of the modern flora, and are preserved only in the fossil record. Therefore, fossils provide vital evidence, unavailable otherwise, for understanding the origins of modern plant structure and for reconstructing the patterns of structural changes through time that have produced the morphological diversity that characterizes modern vegetation.

\section{At macroevolutionary scales, ecological crisis is the driver and natural selection the filter of evolution}

Of equal importance for documenting structural change through time, the fossil record provides convincing evidence for the fundamental processes that underlie plant evolution. While evolution traditionally has been explained within the context of classical and population genetics, as early as the 1970 s tests of those traditional evolutionary hypotheses using paleontological data began to reveal dramatically different patterns of change than those predicted by genetics-based evolutionary theory (Eldredge \& Gould 1977). Population genetics theory predicts that natural selection is the driving force for evolutionary change and that such selective forces are most impactful within well-established ecosystems. Contradicting these predictions, the paleontological record reveals that the most rapid evolutionary diversifications occur immediately after biological catastrophes, wherein extinction has dramatically reduced biotic selective pressures, and has opened up vast swaths of ecological space for colonization by new species (Valentine \& Campbell 1975; Douglas \& Valentine 2013). Both macroevolutionary theory (e.g., Bateman 1999) and paleontologically established patterns of evolutionary change indicate that the reestablishment of complex plant communities leads to long periods of evolutionary stasis, rather than to increased selection-driven evolutionary modification (DiMichele et al. 1987; DiMichele \& Phillips 1996). Therefore, the fossil record reveals that evolutionary diversification is the least rapid when genetically-based evolutionary theory predicts it should reach its highest rates. Likewise, within this paleontologically-sanctioned context natural selection acts as a filter to remove maladapted phenotypes, not as a driving force in evolution (Rothwell 1987).

\section{The evo-devo paradigm and the role of morphology}

A growing appreciation for the role of development in evolution, whose understanding has been dramatically elevated by the advent of developmental molecular biology, has fostered a new and radically different perception of evolutionary process (Langdale \& Harrison 2008; Cronk 2009). Within this context, we now recognize that the genome represents the equivalent of a developmental software program, and it is that program that evolves through time (Rothwell et al. 2014). For each organism, the genetic program is implemented through the processes of development, and the mature organism represents the cumulative structural evidence for both the activity of regulatory genes and, of equal importance, for the developmental processes themselves. Therefore, when various factors lead to changes in the developmental program, there are predictable changes in the resulting organisms (Gould 1977). 
For example, the origin of branching in the sporophyte phase of the embryophyte life cycle was the seminal event leading to the evolution of vascular plants (Kenrick and Crane 1997). That change may be hypothesized to have resulted from the prolongation of the time during which the sporophyte underwent apical growth before going through a transition to reproductive growth and the production of terminal sporangia (Tomescu et al. 2014; Rothwell et al. 2014). According to this hypothesis, a change in developmental regulation in young embryophyte sporophytes resulted in the origin of potentially indeterminate growth from an apical meristem which, in turn, allowed for branching. Evidence in support of this hypothesis is provided by apogamous sporophytes of the living moss Physcomitrella patens, wherein the combination of gene silencing and auxin transport inhibition produces a comparable heterochronic change that results in an elongated and branched axial sporophyte body (see Tomescu et al. 2014 for a detailed review).

Because phenotypes are the direct result of development under the influence of genetic regulation, the wedding of paleontology (i.e., phenotypes through time) with regulatory genetics (i.e., genomic changes leading to structural changes) provides a framework for understanding both evolutionary pattern and process. Within this context, the developmental underpinnings of morphology take on a much more central role in understanding both the patterns and processes of plant evolution, and the fossil record provides the only access to direct evidence of that evolution.

\section{Developmental regulation is modular and hierarchical}

Throughout the ontogeny of an organism, developmental regulation is a complex, dynamic network of interactions between proteins, hormones, small RNAs, etc. An important feature of this network is that the strength and duration of interactions between its components differ, and these differences circumscribe regulatory modules. Such modules comprise networks of interactions that are tightly integrated internally, on one hand, and on the other hand are independent from, or more loosely integrated with, other such networks of interactions. In many cases, integration of regulatory modules involves hierarchical interactions, but individual modules can also be turned on and off independently of other such modules.

In an experimental study of vascular cambial growth in Ficus, Lev-Yadun (1994) demonstrated that girdling induces transient production of wood in which rays develop normally but the axial system exhibits dramatically altered anatomy, differentiating into isodiametric parenchyma. Without addressing directly specific genetic and molecular factors that control development, this example illustrates both modularity and hierarchy of modules in developmental regulation. The different effects that the girdling treatment had on the anatomy of the two systems of secondary xylem - radial (rays) and axial (tracheary elements, xylem parenchyma, fibers) - indicates that their development is controlled independently, reflecting distinct regulatory modules, at least of the level of patterns of cell division and differentiation. Girdling, on the other hand, did not directly affect the three-dimensional organization of secondary xylem (into two distinct systems), which suggests that this organization is controlled at a different hierarchical level of developmental regulation.

The modularity of developmental regulatory networks allows for broad variation in the way developmental programs are assembled over evolutionary time, with different developmental outcomes in different organisms. The roots of variability reside in (1) the degree of integration of the modules, which can be more or less tightly integrated (or more or less dependent on each other) in more or less hierarchical ways, and in (2) the combinatorial nature of the activation (or lack thereof) of different modules at different stages in ontogeny. Together, these sources of variation allow for a vast amount of potential diversity in developmental programs (i.e., different modules being turned on or off, separately or driving downstream cascades). Such potential provides the raw material for selection and, ultimately, morphological evolution. Thus, for example, analyses of morphological evolution across plant phylogeny have assembled data that indicate different pathways of accretion of complexity in different 
lineages (Bonacorsi \& Leslie 2019; Crepet \& Niklas 2019), and support hypotheses about the evolution of morphological complexity as a mosaic of features combined in different ways and assembled in different sequences in different major lineages (Tomescu \& Groover 2019; Crepet \& Niklas 2019).

\section{Structural fingerprints provide evidence for the deployment of regulatory modules across phylogeny and time}

Many anatomical and morphological features seen in organisms bear witness to the activity and, sometimes, interactions of specific regulatory modules. Such anatomical or morphological features that are the product of a specific developmental process represent structural fingerprints of the activity of regulatory modules that control that process. In other words, studying morphology can teach us about developmental regulation. In plants, specifically, identification of such fingerprints is facilitated by the fact that the position of cells is largely fixed; cells are attached to each other by their walls, in more-orless the position in which they arise by cell division. As a result, the relative arrangement of cells records sequences of cell division, allowing for more detailed reconstruction of developmental processes. Such is, for instance, the easily distinguishable patterning of merophytes derived from immediate derivatives of the apical cell and arranged around the latter on a fern shoot apical meristem; or the arrangement of cells in cross sections of wood that records the sequence of past periclinal and anticlinal divisions in the cambial initials.

Although gene regulation, resulting in structural features of plants, is a transitory process not available for direct examination from fossils, these features - structural fingerprints - do accurately reflect the regulatory genetics by which they were produced. Therefore, when structural fingerprints are identified in fossils, they can be employed to infer the specific regulatory programs by which they developed (Rothwell \& Lev-Yadun 2005). Because in living plants we can tie these fingerprints to specific, detailed regulatory programs, we can circumscribe the exact nature of regulatory modules and of interactions within and between modules that generate the structural fingerprints. If compared between extant plant lineages, this type of information can reveal the degree of variation in the structure and interaction of regulatory modules that characterize different lineages. Studies that integrate structural fingerprints and molecular-genetic regulation in living plants allow us to infer the same relationships between gene regulation and structure in extinct plants. This opens up a whole new window onto the evolution of development, by allowing us to trace the presence of regulatory processes and the activity of specific regulatory modules in phylogenetic space and evolutionary time. In other words, this methodology allows us to connect the regulatory genetics of living forms to their long-extinct ancestors and precursors (or, at least, to form hypotheses about such connections) within an empirically-based framework (e.g., Tomescu et al. 2017).

For example, if a particular regulatory pathway is shared by sister clades, then we can hypothesize that they share a common developmental tool kit which has been inherited from a common ancestor that possessed that tool kit (Rothwell et al. 2014). Those hypotheses can then be tested by searching for the structural fingerprint of those tool kits in specimens of the common ancestor (or extinct sister group) of the two clades. An example of that sort of hypothesis test is presented below for the role of polar auxin regulation in the development of vascular tissue.

\section{A quintessential structural fingerprint and its implications}

Polar auxin regulation of both primary and secondary vascular tissue production is well established among vascular plants, wherein it evolved increasing sophistication at successively more distal levels of their phylogeny (Sachs \& Cohen 1982; Cooke et al. 2002; Rothwell et al. 2014). During secondary growth from a vascular cambium, polar auxin regulation of cell positioning and growth results in characteristic circular patterns in the secondary xylem. These patterns consist of swirls of tracheary elements positioned upstream of locations where polar auxin flow in the cambium has been impeded by 
obstructions, such as axillary buds and lateral branches (Sachs \& Cohen 1982; Hejnowicz \& Kurczyńska 1987; Lev-Yadun \& Aloni 1990), thus representing anatomical fingerprints for polar auxin regulation of secondary xylem patterning. Such circular patterns were the first structural fingerprints to be recognized in fossil plants (Rothwell \& Lev-Yadun 2005; Rothwell et al. 2014).

Because xylem has excellent fossilization potential, wood anatomy is among the most common sources of data in the plant fossil record. Herein, swirls of tracheary elements provide powerful evidence (1) for polar auxin transport as a regulatory mechanism of vascular cambial growth shared among several major plant clades (Rothwell et al. 2008), and(2) for the antiquity of polar auxin regulation in secondary growth. This demonstrated shared mechanism suggests that at least some of the basic regulatory programs that control secondary growth may have been part of a developmental toolkit shared among all euphyllophytes, or even all tracheophytes (Hoffman \& Tomescu 2013; Tomescu \& Groover 2019). The same structural fingerprint identified in the rooting structures (rhizomorphs) of arborescent lepidodendralean lycopsids demonstrated that these positively gravitropic axes have acropetal auxin transport, unlike the shoots to which they are homologous, and similar, instead, to other rooting structures with different homologies (Sanders et al. 2011; Rothwell \& Tomescu 2018). In turn, this shared directionality of polar auxin transport implies that acropetal auxin flow transcends organ identity and is more tightly linked to positively gravitropic axes, independent of their homology (Tomescu \& Matsunaga 2019) - whether they be roots (as in most extant tracheophytes), modified shoots (in isotalean and lepidodendrid lycophyte rhizomorphs, and in drepanophycalean lycophyte rooting axes), rhizomorphs (in Selaginella), or simple undifferentiated axes (in zosterophylls).

\section{Combining structural fingerprints}

By the beginning of the Silurian (444 Ma ago), basal members of the clade characterized by branched sporophytes (i.e., polysorangiophytes, including all vascular plants) had emerged (Salamon et al. 2018) out of a plexus of basal embryophytes whose earliest bryophyte-grade representatives go as far back as the Middle Ordovician (468 Ma ago; Rubinstein et al. 2010). If trilete spores are, indeed, exclusively characteristic of vascular plants and not of all the embryophytes, as has been proposed by Steemans et al (2009), then vascular plants and, by extension, polysporangiophytes may have evolved as early as $455 \mathrm{Ma}$ ago, around the beginning of the Late Ordovician (Wellman \& Strother 2015; Rubinstein \& Vajda 2019). Direct information on these plants is available exclusively from fossils, which provide multiple structural fingerprints that, combined, allow us to reconstruct the morphology and life history of these tracheophyte ancestors.

Early polysporangiophytes had diminutive sporophytes that were only little more than branched versions of bryophyte-grade sporophytes (Tomescu et al. 2014). The small size of the sporophytes is immediately apparent in the fossils (Boyce 2008; Salamon et al. 2018). The branching of these sporophytes indicates that they grew from apical meristems, but their diminutive size, scant branching, and presence of sporangia terminating all branches (e.g., Tomescu et al. 2014; Salamon et al. 2018) indicate that their meristematic growth was determinate. The small size of these sporophytes also supports the hypothesis that they were nutritionally dependent on the gametophytes (Boyce 2008), like the sporophytes of bryophytes. The dependence of sporophytes is also consistent with the inference that their growth was determinate. Whether the earliest polysporangiophyte sporophytes possessed tracheid-based water-conducting tissues is less clear, as the oldest tracheids discovered to date are 424 Ma old (Edwards \& Davies 1976). This is close to the (slightly older, ca. $432 \mathrm{Ma}$ ) age of the oldest known sporophytes that reached sizes consistent with physiological independence (Libertín et al. 2018).

The gametophytes that supported these diminutive sporophytes were most likely thalloid, like those of hornworts and liverworts and unlike those of younger, Devonian polysporangiophytes such as Aglaophyton, Rhynia, Horneophyton or Nothia (Taylor et al. 2005). This inference is based on the thalloid form of fossils found associated with (but not attached to) branched sporophytes in Silurian 
layers at multiple locations (Edwards 1979; Strother 2010), and on structural and chemical evidence that some of these fossils are plants (Tomescu \& Rothwell 2006; Tomescu et al. 2009; Tomescu et al. 2010).

\section{Echoes of modularity}

\subsection{The leaves of ferns and seed plants}

The Euphyllophytina is the largest and most diverse of the two major clades of living vascular plants, and is represented in the modern flora by flowering plants, gymnosperms, ferns, Equisetum, and psilotophytes (i.e., Psilotum and Tmesipteris). All of the extant euphyllophytes (with the possible exception of psilotophytes; Tomescu 2011) show stem-leaf-root organography in their vegetative sporophyte. Systematic analyses of living species have inferred that this derived organography has evolved only once among euphyllophytes (Schneider et al. 2002). However, the basal grade of fossil euphyllophytes, referred to as trimerophytes, all lack leaves. This absence of leaves in the basal-most lineages of the clade, coupled with the position of these lineages within the topology of euphyllophyte phylogeny (Kenrick \& Crane 1997; Rothwell 1999), and fossil evidence for the evolution of euphyllophyte leaves (Sanders et al. 2009; Boyce \& Knoll 2002), provide compelling evidence that leaves evolved independently, in parallel, in several different euphyllophyte lineages from such leafless trimerophytes (Tomescu 2009). Thus, the leaves of different euphyllophyte clades that appear to be homologous to neontologists, actually result from parallel evolution (Rothwell 1999; Boyce \& Knoll 2002; Boyce 2005; Sanders et al. 2009; Tomescu 2009). This is one of the most compelling examples of fossils and morphology allowing for the recognition of analogy (or homoplasy; similar characters in two groups that evolved independently by parallel or convergent evolution) and its distinction from homology (i.e., characters in two groups that are inherited from a common ancestor that had those characters).

The two main structural changes that have led to the evolution of leaves from leafless trimerophyte axes are (1) the change from indeterminate to determinate growth, and (2) the origin of abaxial-adaxial patterning in the transition from radial to bilateral growth (Sanders et al. 2007). Living euphyllophyte leaves have both determinate growth and bilateral (abaxial-adaxial) polarity, each of these properties being controlled by specific regulatory modules. In both ferns and angiosperms meristematic activity at the shoot apex is maintained by class I KNOX genes and LFY. These genes are also responsible for proliferative growth in the leaves of both ferns and angiosperms (e.g., compound leaves) (Tomescu 2009; Harrison \& Morris 2017; Maugarny-Calès \& Laufs 2018; Plackett et al. 2018). Thus, determinacy of growth in leaves reflects the evolution of regulatory programs that repress these genes, such as the ARP group genes that repress KNOX I gene activity. In all euphyllophytes, adaxialabaxial polarity (sometimes referred to as dorsiventral polarity) results from the expression of, and interactions between, class III HD-ZIP genes (promoters of adaxial identity) and KANADI genes (promoters of abaxial identity) (Floyd \& Bowman 2006; Vasco et al. 2016; Zumajo-Cardona et al. 2019). Adaxial-abaxial polarity is reflected in the flattened morphology of leaves and, even in the absence of this morphology, can be ascertained based on the bilateral patterning of the vascular tissues that supply these lateral appendages.

Using these structural fingerprints - that reflect determinate growth and adaxial-abaxial polarity -, and querying the fossil record of early ferns and seed plants, Sanders et al. (2009) demonstrated that whereas seed plants evolved determinate growth before adaxial-abaxial polarity in the leaves, in filicalean fern leaves evolution of adaxial-abaxial polarity preceded determinacy. Aside from supporting hypotheses of independent evolution of leaves in ferns and seed plants, which followed different trajectories in terms of sequence of character evolution, this is consistent with a modular nature of the regulators of leaf determinacy and adaxial-abaxial polarity, which allows for independence in their deployment and activity. Thus, structural fingerprints for developmental mechanisms preserved in fossils provides evidence for the modular nature of specific aspects of developmental regulation. 
Venation is an additional facet of leaf (or pinnule) structure that reveals structural fingerprints of the meristematic activities which generated it. Tracking the deployment of these activities across plant phylogeny and the fossil record reveals further evidence for the parallel evolution of leaves within Euphyllophytina. The paleontological record documents leaf evolution within several clades of Paleozoic euphyllophytes and provides direct evidence for parallel changes in pinnule structure and leaf venation in each (Boyce \& Knoll 2002). Specifically, the fossil record demonstrates that in each of at least four clades (i.e., seed plants, ferns, sphenopsids, and progymnosperms) the most ancient representatives produced ultimate units (i.e., pinnules) that had linear laminar segments with marginal vein endings, and that successively more recent representatives progressed through parallel modifications to (1) divergent venation with marginal vein endings; (2) convergent venation with marginal vein endings; (3) reticulate venation with marginal vein endings; and (4) reticulate venation with internal vein endings (summarized by Rothwell et al. 2014). Although the complete series of structural/meristematic modifications was achieved in only ferns and seed plants, these parallel evolutionary trajectories of leaf venation represent structural fingerprints for a succession of parallel changes in the meristems that contributed to the evolution of euphyllophyte leaves (or their ultimate segments in the case of compound leaves) in all four clades.

\subsection{Secondary growth}

The modularity of developmental regulation takes on a much broader scope if we consider the evolution of secondary growth from a vascular cambium and the diversity of modes of secondary growth that have arisen among tracheophytes. One of the major unanswered questions regarding the evolution of secondary growth is whether vascular cambial growth evolved independently in different tracheophyte lineages or only once, at the base of the clade. The traditional view on this issue, based on records of secondary growth in multiple lineages that go back to the Middle Devonian, has been that vascular cambial growth originated independently in the different lineages. This perspective has its roots in the perceptions that (i) the first occurrences of secondary growth in the different lineages are much younger than the origin of tracheophytes; and (ii) that the anatomy of secondary tissues shows significant differences between major lineages (Cichan, 1985).

The traditional view on the evolution of vascular cambial growth is currently reshaped by evidence coming from two directions. First, there is the realization that some mechanisms regulating cambial growth are shared among major tracheophyte lineages (spanning the lycopsids and the euphyllophytes: lepidodendrales, sphenopsids, progymnosperms, and spermatophytes) (Rothwell et al. 2008; Sanders et al. 2011). Second, accumulating discoveries (Gerrienne et al. 2011; Hoffman \& Tomescu 2013; Strullu-Derrien et al. 2014; Gensel 2018) point to much earlier origins of vascular cambial growth than previously thought, at least in the euphyllophyte clade. Together, these lines of evidence suggest that mechanisms regulating secondary growth may have become part of the euphyllophyte developmental toolkit very early in the evolution of the clade. In turn, this possibility begs the question: could these mechanisms have originated in a common ancestor of euphyllophytes, or even the common ancestor of euphyllophytes and lycopsids?

To begin answering this question, Tomescu and Groover (2019) have proposed an updated perspective that approaches vascular cambial growth as a complex developmental process of modular nature. In this perspective, the diverse anatomies of secondary growth seen in different extinct lineages (i.e., modes of secondary growth) reflect a mosaic pattern of expression of distinct, more-or-less independent developmental regulatory modules that are individually responsible for different component processes that comprise secondary growth. This perspective generates hypotheses that can be tested experimentally, in principle, by altering the activity of different proposed regulatory modules.

Importantly, the activity of the different regulatory modules proposed by Tomescu and Groover can be recognized based on specific anatomical fingerprints that are preserved in the wood (secondary 
xylem) of plants, including fossil plants. The presence or absence of these fingerprints in the wood of different lineages are evidence that the regulatory modules are deployed differently among different lineages, living and extinct - some are shared among multiple lineages, while others are apomorphic for distinct lineages. Thus, information preserved in fossils and an understanding of structural fingerprints for developmental processes, combined in the context of an evolutionary-developmental perspective that is rooted in modularity of developmental regulatory mechanisms, can contribute to the construction of testable hypotheses about the evolutionary origins of secondary growth.

\section{Modularity and hierarchy}

Within the paradigm of modularity in development, information preserved in fossils and recognized as structural fingerprints for specific developmental regulators can also lead to inferences of hierarchy in the deployment of regulatory modules. An example is the case of the regulatory programs that underlie the reproductive morphologies of living and extinct sphenopsids of the family Equisetaceae. Represented today solely by the genus Equisetum, this clade represents an excellent example of a long phylogenetic branch wherein homology issues can only be resolved by querying the rich fossil record of the group (Elgorriaga et al. 2018). Furthermore, the strobilus of Equisetum, has been for a long time a puzzle in terms of homology and morphological evolution, especially when considered in the context of the fossil record of the group (Cuneo \& Escapa 2006). The stalemate was rooted in the seemingly contradictory homology implications of different reproductive morphologies present in the group, when considered from the perspective of the shoot as an alternation of nodes and internodes. In brief, the frustrating question was: Are the sporangium-bearing appendages (sporangiophores) attached at nodes or along internodes? For the Equisetum strobilus, this question boiled down to whether it represents multiple nodes, each bearing a whorl of sporangiophores, or a single terminal internode with multiple sporangiophore whorls attached along it.

Studies of development in living Equisetum show that shoots grow as a result of the combined activity of the apical meristem, which generates phytomers, and intercalary meristems, which are responsible for elongation of the internode in each phytomer. This suggested that an emphasis on the phytomeric structure of the shoot, rather than the node-internode alternation, may provide a more appropriate paradigm within which to understand homology in the Equisetum strobilus and, more broadly, in equisetacean reproductive morphology (Tomescu et al. 2017). At the same time, current understanding of plant developmental regulation indicates that meristems of all types are equivalent in their fundamental capacities, including the capacity to transition to reproductive growth, and that the regulatory programs effecting this transition are shared broadly among tracheophytes. Together, these observations lead to the hypothesis that in equisetaceans the switch to a reproductive developmental program happens in the intercalary meristems responsible for internode elongation and, as a result, sporangiophore whorls are produced along the internodes of fertile phytomers.

The hypothesis of reproductive growth in internode intercalary meristems generates predictions about morphological patterns produced by this mode of development that can be used as structural fingerprints, which are recognized in the equisetacean fossil record, confirming the presence of intercalary reproductive growth, the only instance of its kind known in plants. This confirmation provides an updated framework for understanding the origin of the Equisetum strobilus and other reproductive morphologies present among equisetacean sphenophytes. These different morphologies are best explained as resulting from deployment of independent regulatory modules expressed in a hierarchic sequence. The regulatory modules (1) turn on reproductive growth in the phytomer, (2) lead to determinate apical growth, and (3) repress node-internode differentiation and intercalary meristematic activity in the fertile phytomers, respectively.

The view of the Equisetum strobilus as a determinate stack of fertile phytomers without nodeinternode identity that each differentiate into a sporangiopore whorl, offers solutions for the homology 
and evolution of the equisetalean sporangiophore, relevant to the deep history of the sphenophyte lineage. If sporangiophore development is independent of nodal/internodal identity, the sporangiophore must have followed an evolutionary trajectory independent of the evolution of nodeinternode organization in shoots and whose beginnings may have pre-dated node-internode organization. Thus, the sphenopsid sporangiophore could represent a conserved regulatory module originally responsible for the development of fertile lateral branching systems, a module that underwent its own evolution that included heterotopic change, from expression along undifferentiated axes, to expression on specialized shoot segments, the internodes.

From an epistemic standpoint, this case study demonstrates how a hypothesis generated by data from living plants was tested and confirmed using data from the fossil record. In turn, this provided a framework for subsequent hypotheses that included data from living Equisetum and fossil plants, to offer a novel explanation, involving a hierarchy of regulatory modules, of the origin of the Equisetum strobilus and other reproductive morphologies of fossil sphenophytes. This updated perspective on the Equisetum strobilus generates further hypotheses about evolution and the deep fossil record, explaining the origin and evolution of the sphenophyte sporangiophore, all of which are possible only because evolutionary data have been preserved in the fossil record.

\section{Conclusions}

The paleontological record, which provides the best evidence for evolutionary pattern, can now also be used to address fundamental questions about evolutionary process, through the use of structural fingerprints for plant development. Studies applying the epistemic framework of this paleoevo-devo perspective and methodology illuminate our understanding of how evolution proceeds by successive modifications of plant development, which are controlled, in turn, by the activities of regulatory genes and growth regulators. This approach further clarifies that developmental regulation of plant growth is both modular and hierarchical. When coupled with another based of knowledge informed by the fossil record - our understanding of the overall pattern of plant phylogeny -, characterization of such developmental modules, of the lineages in which they have been deployed, and of the order in which they have accumulated in divergent lineages, provide a backbone for identifying both the specific processes and the patterns by which evolution has proceeded. Continued exploration of three directions - (1) the composition, structure, and functioning of gene regulatory networks that underpin all aspects of the morphological variety seen across the diverse extant plant lineages; (2) the distinct morphological and anatomical signatures of regulatory modules (i.e., structural fingerprints) that are shared among multiple extant lineages; and (3) the occurrence of such fingerprints in the fossil record, across geologic time and phylogenetic space - will lead to deeper and more meaningful integration of data from the fossil record in the overall tapestry of the evolution of development throughout the history of plant life.

\section{References}

Bateman, R. M. (1999). Integrating molecular and morphological evidence of evolutionary radiations. Molecular systematics and plant evolution. P. M. Hollingsworth, R. M. Bateman and R. J. Gornall. London, UK, Taylor \& Francis: 432-471.

Bonacorsi, N. K. and A. B. Leslie (2019). Sporangium position, branching architecture, and the evolution of reproductive morphology in Devonian plants. International Journal of Plant Sciences (Int. J. Plant Sci.) 180: 493-503.

Boyce, C.K. (2005). Patterns of segregation and convergence in the evolution of fern and seed plant leaf morphologies. Paleobiology 31: 117- 140. 
Boyce, C.K. (2008). How green was Cooksonia? The importance of size in understanding the early evolution of physiology in the vascular plant lineage. Paleobiology 34: 179- 194.

Boyce, C.K. and A.H. Knoll. (2002). Evolution of developmental potential and the multiple independent origins of leaves in Paleozoic vascular plants. Paleobiology 28: 70 - 100.

Cichan, M.A. (1985). Vascular cambium and wood development in Carboniferous plants. II. Sphenophyllum plurifoliatum Williamson and Scott (Sphenophyllales). Botanical Gazette 146: 395403.

Cooke, T.J., D.B Poli, A.E. Sztein and J.D. Cohen. (2002). Evolutionary patterns in auxin action. Plant Molecular Biology 49: 319-338.

Crepet, W. L. and K. J. Niklas (2019). The evolution of early vascular plant complexity. International Journal of Plant Sciences (Int. J. Plant Sci.) 180: 800-810.

Cronk, Q.C.B. (2009). The molecular organography of plants. Oxford University Press, Oxford, UK.

Cuneo, N. R. and I.H. Escapa (2006). The equisetalean genus Cruciaetheca nov. from the Lower Permian of Patagonia, Argentina. International Journal of Plant Sciences 167: 167-177.

DiMichele, W.A. and T.L. Phillips. (1996). Climate change, plant extinctions and vegetational recovery during the Middle-Late Pennsylvanian Transition: The case of tropical peat-forming environments in North America. In: Hart, M.B., ed., Biotic recovery from mass extinction events. Geological Society of America Special Publication 102: 201-221.

DiMichele, W.A., T.L. Phillips and R.G Olmstead. (1987). Opportunistic evolution: Abiotic environmental stress and the fossil record of plants. Review of Palaeobotany and Palynology 50: 151-187.

Douglas, E.H. and J. W. Valentine. (2013). The Cambrian explosion: the construction of animal biodiversity. Roberts and Co., Greenwood Village, Colorado, USA.

Edwards, D. (1979). A Late Silurian flora from the lower Old Red Sandstone of South-West Dyfed. Palaeontology 22: 23-52.

Edwards, D. and E. C. W. Davies (1976). Oldest recorded in situ tracheids. Nature 263: 494-495.

Eldredge, N. and S.J. Gould. (1977). Punctuated equilibria: the tempo and mode of evolution reconsidered. Paleobiology 3: 115-151.

Elgorriaga, A., I. H. Escapa, et al. (2018). Origin of Equisetum: evolution of horsetails (Equisetales) within the major euphyllophyte clade Sphenopsida. American Journal of Botany 105: 1286-1303.

Floyd, S. K. and J. L. Bowman (2006). Distinct developmental mechanisms reflect the independent origins of leaves in vascular plants. Current Biology 16: 1911-1917.

Gensel, P.G. (2018). Early Devonian woody plants and implications for the early evolution of vascular cambia. In: Krings, M., C.J. Harper, N.R. Cúneo and G.W. Rothwell, eds., Transformative Paleobotany. Academic Press, London, UK.

Gerrienne, P., P. G. Gensel, et al. (2011). A simple type of wood in two Early Devonian plants. Nature 333: 837.

Gould, S.J. (1977). Ontogeny and phylogeny. Belknap Press/Harvard University Press, Cambridge, MA, USA.

Harrison, C. J. and J. L. Morris (2017). The origin and early evolution of vascular plant shoots and leaves. Philosophical Transactions of the Royal Society B: Biological Sciences 373: 20160496.

Hejnowicz, Z., Kurczyńska, E.U. (1987). Occurrence of circular vessels above axillary buds in stems of woody plants. Acta Societatis Botanicorum Poloniae 56: 415-419.

Hoffman and Tomescu. (2013). An early origin of secondary growth: Franhueberia gerriennei gen. et sp. nov. from the Lower Devonian of Gaspé (Quebec, Canada). American Journal of Botany 100: 754763.

Kenrick, P. and P.R. Crane. (1997). The origin and early diversification of plants on land: a cladistics study. Smithsonian Institution Press, Washington, DC. 
Langdale, J. A. and C. J. Harrison. (2008). Developmental transitions during the evolution of plant form. Evolving pathways. Key themes in evolutionary developmental biology. A. Minelli and G. Fusco. Cambridge, UK, Cambridge University Press: 299-315.

Lev-Yadun, S. (1994). Experimental evidence for the autonomy of ray differentiation in Ficus sycomorus L. New Phytologist 126: 499-504.

Lev-Yadun, S. and R. Aloni. (1990). Vascular differentiation in branch junctions of trees: circular patterns and functional significance. Trees 4: 49-54.

Libertín, M., J. Kvacek, et al. (2018). Sporophytes of polysporangiate land plants from the early Silurian period may have been photosynthetically autonomous. Nature Plants 4: 269-271.

Maugarny-Cales, A. and P. Laufs. (2018). Getting leaves into shape: a molecular, cellular, environmental and evolutionary view. Development 145: dev161646.

Plackett, A. R. G., S. J. Conway, et al. (2018). LEAFY maintains apical stem cell activity during shoot development in the fern Ceratopteris richardii. eLife 7: e39625.

Rothwell, G.W. (1987). The role of development in plant phylogeny: a paleobotanical perspective. Review of Palaeobotany and Palynology 50: 97-114.

Rothwell, G.W. (1999). Fossils and ferns in the resolution of land plant phylogeny. Botanical Review 65: 188-217.

Rothwell, G.W. and S. Lev-Yadun. (2005). Evidence of polar auxin flow in 375 million-year-old fossil wood. American Journal of Botany 92: 903-906.

Rothwell, G.W., H. Sanders, S.E. Wyatt and S. Lev-Yadun. (2008). A fossil record for growth regulation: the role of auxin in wood evolution. Annals of the Missouri Botanical Garden 95: $121-134$.

Rothwell, G.W., Wyatt, S.E. and A.M.F. Tomescu. (2014.) Plant evolution the interface of paleontology and developmental biology: an organism-centered paradigm. American Journal of Botany 101: 899913.

Rothwell, G.W., \& A.M.F. Tomescu. (2018). Structural fingerprints of development at the intersection of evolutionary developmental biology and the fossil record. Evolutionary-Developmental Biology Reference Guide; Plants. Springer Verlag Books. In: Nuño de la Rosa L, Müller G, eds. Evolutionary developmental biology - a reference guide. Basel, Switzerland: Springer Nature, doi: 10.1007/978-3319-33038-9_169-1

Rubinstein, C. V., P. Gerrienne, et al. (2010). Early Middle Ordovician evidence for land plants in Argentina (eastern Gondwana). New Phytologist 188: 365-369.

Rubinstein, C. V. and V. Vajda (2019). Baltica cradle of early land plants? Oldest record of trilete spores and diverse cryptospore assemblages; evidence from Ordovician successions of Sweden. GFF 2019. doi: 10.1080/11035897.2019.1636860

Sachs, T. and D. Cohen. (1982). Circular vessels and the control of vascular differentiation in plants. Differentiation 21: 22-26.

Salamon, M. A., P. Gerrienne, et al. (2018). Putative Late Ordovician land plants. New Phytologist 218: 1305-1309.

Sanders, H., G.W. Rothwell and S.E. Wyatt. (2007). Paleontological context for the developmental mechanisms of evolution. International Journal of Plant Sciences 168: $719-728$.

Sanders, H., G.W. Rothwell and S.E. Wyatt. (2009). Key morphological alterations in the evolution of leaves. International Journal of Plant Sciences 170: 860 - 868.

Sanders, H., G.W. Rothwell and S.E. Wyatt. (2011) Parallel evolution of auxin regulation in rooting systems. Plant Systematics and Evolution 291: 221 - 225.

Schneider, H., K. M. Pryer, et al. (2002). Evolution of vascular plant body plans: a phylogenetic perspective. Developmental genetics and plant evolution. Q. C. B. Cronk, R. M. Bateman and J. A. Hawkins. London, Taylor \& Francis: 330-364. 
Steemans, P., A. Le Herisse, et al. (2009). Origin and radiation of the earliest vascular land plants. Science 324: 353.

Strother, P. K. (2010). Thalloid carbonaceous incrustations and the asynchronous evolution of embryophyte characters during the Early Paleozoic. International Journal of Coal Geology 83: 154161.

Strullu-Derrien, C., P. Kenrick, et al. (2014). The earliest fossil wood and its hydraulic properties documented in c. 407-million-year-old fossils using synchrotron microtomography. Botanical Journal of the Linnean Society 175: 423-437.

Taylor, T. N., H. Kerp, et al. (2005). Life history biology of early land plants: deciphering the gametophyte phase. Proceedings of the National Academy of Sciences 102: 5892-5897.

Tomescu, A.M.F. (2009). Megaphylls, microphylls and the evolution of leaf development. Trends in Plant Science 14: 5-12.

Tomescu, A.M.F. (2011). The sporophytes of seed-free vascular plants-Major vegetative developmental features and molecular genetic pathways. In H. Fernandez, A. Kumar, and M. A. Revilla [eds.], Working with ferns-Issues and applications, 67-94. Springer, New York, New York, USA.

Tomescu, A.M.F., L. M. Pratt , G. W. Rothwell , P. K. Strother , and G.C. Nadon. (2009). Carbon isotopes support the presence of extensive land floras pre-dating the origin of vascular plants. Palaeogeography, Palaeoclimatology, Palaeoecology 283: 46 - 59.

Tomescu, A.M.F., R. W. Tate, N. G. Mack, and V. J. Calder. (2010). Simulating fossilization to resolve the taxonomic affinities of thalloid fossils in Early Silurian (ca. $425 \mathrm{Ma}$ ) terrestrial assemblages. In T. H. Nash III et al. [eds.], Biology of lichens-Symbiosis, ecology, environmental monitoring, systematics and cyber applications, 183-189. J. Cramer/Gebrueder Borntraeger Verlagsbuchhandlung, Stuttgart, Germany.

Tomescu, A.M.F., I.H. Escapa, G.W. Rothwell, A. Elgorriaga and N.R. Cuneo. (2017). Developmental programmes in the evolution of Equisetum reproductive morphology: a hierarchical modularity hypothesis. Annals of Botany 119: 489-505.

Tomescu, A. M. F. and A. T. Groover. (2019). Mosaic modularity: an updated perspective and research agenda for the evolution of vascular cambial growth. New Phytologist 222: 1719-1735.

Tomescu, A. M. F. and K. K. S. Matsunaga. (2019). Polar auxin transport and plant sporophyte body plans. Reference Module in Life Sciences Evolutionary developmental biology - a reference guide. Basel, Switzerland: Springer Nature, doi: 10.1016/B978-0-12-809633-8.20905-9

Tomescu, A.M.F. and G.W. Rothwell. (2006). Wetlands before tracheophytes: thalloid terrestrial communities of the Early Silurian Passage Creek biota (Virginia). Wetlands through time. S. F. Greb and W. A. DiMichele (eds), Geological Society of America. 399: 41-56.

Tomescu, A.M.F., S.E. Wyatt, M. Hasebe and G.W. Rothwell. (2014). Early evolution of the vascular plant body plan - The missing mechanisms. Current Opinion in Plant Biology 17: 126-136.

Valentine, J.W. and C.A. Campbell. (1975). Genetic regulation and the fossil record. American Scientist 63: 673-680.

Vasco, A., Smalls, T.L., Graham, S.W., et al., (2016). Challenging the paradigms of leaf evolution: class III 1045 HD-Zips in ferns and lycophytes. New Phytologist 212: 745-758.

Wellman, C.H. and P.K. Strother. (2015). The terrestrial biota prior to the origin of land plants (embryophytes): a review of the evidence. Palaeontology 58: 601-627.

Zumajo-Cardona, C., A. Vasco, et al. (2019). The evolution of the KANADI gene family and leaf development in lycophytes and ferns. Plants 8: 313. 